\title{
Review of Grounding Grids Corrosion Diagnosis
}

\author{
Wenguang Chen, Ruyu Bi, Jian Wang, and Hougui Chen
}

\begin{abstract}
This article introduces several existing detection methods of grounding grid corrosion, such as the method based on electricity network, the method based on electromagnetic theory and the electrochemical-based method. Subsequently, this article provides analysis of these three methods, including analysis and comparison of their advantages and disadvantages. Finally it proposes a new method based on ultrasonic guided wave method and then explores its feasibility and ad-vantages.
\end{abstract}

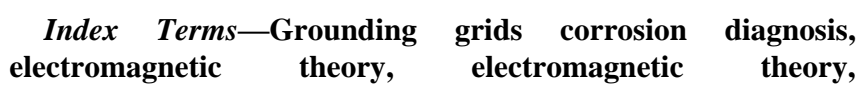
electrochemical method, ultrasonic guided wave.

\section{INTRODUCTION}

The grounding grid is the facilities to ensure the electrical equipment and personal safety. When the grounding grid is defective, the safety of the electrical equipment and the operator will be in danger. The material of substation grounding grid abroad is mostly made of copper material whose corrosion is small and the degree of corrosion can be evaluated by measuring the galvanic current over the deflectors [1]. In China the grounding grid materials are mainly round and flat steel. Due to years of soil erosion and the role of grounding short-circuit current, different part of the grounding grid have varying degrees of corrosion, resulting in the decrease of reliability or even in failure of function. In China, power system accidents caused by grounding grid corrosion occurred frequently. Each accident has brought huge economic losses [2]-[3]. Detection on Grounding grid corrosion can discover the severely corroded areas, taking timely and effective measures to re-duce or eliminate the power system accidents caused by corrosion of the grounding grid. In the past, grounding grid corrosion diagnosis is mainly depended on grounding resistance detection and digging the corroded areas of grounding grids. This method leads to blindness, heavy workload, low efficiency, and is limited by the environment conditions [4-5] Then another method come up is to use power frequency current to detect grid-connected grounding resistance, the potential distribution and the contact voltage, but this method has many difficulties as well, such as the necessity of cutting off power, large current, complex detection work [6].

At present, three kind of method to detect the corrosion of the grounding grid and its breakpoint has been theoretically proposed .They are electrical network analysis [7]-[24], electromagnetic field detection [25]-[31] and electrochemical

Manuscript received October 12, 2012; revised November 20, 2012.This work was supported in part by Wuhan University under Grant 111048638

The authors are with School of Electrical Engineering, Wuhan University 430072 China. (e-mail:chenwenguangc@163.com,923190217@qq.com, 945681253@qq.com,83684787@qq.com). methods [32]-[35].

\section{Method BASEd On ElECtRICAL Network THEORY}

This method has come into practice for a long time. The basic idea of this method: Ignoring the inductance and capacitance of the grounding grid and the grid when the grid is affected by direct current or power-frequency AC current, the grid is an equivalent of pure resistive network. Then the physical problem of grounding grid fault-diagnosis can be turned into electric network problem.

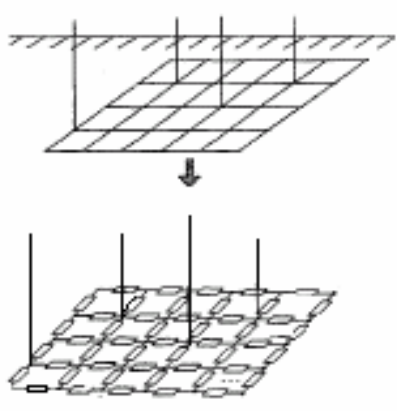

Fig. 1. Grounding grid is equivalent to a purely resistive network

[7]-[9] Zhang Xiaoling and Chen Xianlu, firstly establish the fault diagnosis equation based on the node voltage equations and matrix theory of electricity network. By applying minimum energy principle and optimization techniques as well as other disciplines, they successfully solved the underdetermined equation problem. [10], [16] Liu Qingzhen determined fault by comparing node voltage before and after the fault occurs, and use the neural network methods to achieve intelligent fault-positioning. Jiang Xiubo found the sufficient condition of the existence of grounding grid fault is that the voltage of each node before and after the failure under constant DC power doesn't keep the same. Literatures [11]-[15] propose fixed-point principle based on electrical network theory. This principle requires step by step detection, firstly selecting some measurable nodes used in the field test for fault determination. Then in the surrounding of the preliminary determined point of failure once again makes failure-determine .If the state of fault keeps the same then the failure is determined as real, and vice versa.as the pseudo-fault. Literature [17]-[20] proposed an improved grounding grid fault diagnosis algorithm. This method applied rotation excitation current source position rotation to availability nodes and took multiple-point measuring methods to measure the node voltage. This method brings about a significant increase in the number of equations, establishing an augmented fault diagnosis equation which reflects the nonlinear relationship between changes in resistance of branches and node voltages varying amounts 
affected by a variety of excitation conditions. The equation was solved by iterative, using linear equations to approach the gradual approximation of nonlinear equations. Least squares method was used to find the optimal solution of the optimization method. The number of independent equations can be obtained by finding the distance between the row vector normalized augmented fault diagnosis equation. As a basis for quantitative evaluation of the test method, the number of independent equations can reflect the error on the slip resistance diagnosis using voltage measurement. Literature [21] regards the grounding network as a pure resistance network basing on the circuit and fault diagnosis principles. According to the Tellegen theorem, when the actual ground network topology and ground lead resistance measurement data are available, the grounding grid corrosion diagnosis equations can be established. By solving the diagnostic equation, changes in the value of the grounding grid branch conductor can be founded, and thus determine the grounding grid corrosion.

In general, various methods based on electrical network theory are basically measuring resistance increment of each conductor of the grounding grid (or the node voltage change) as the fault parameters to establish the fault diagnosis equation. Then each of them uses a variety of optimization algorithm to find the solution of the equation. However, the result is mainly the optimal solution, and not necessarily the actual value. To get the actual value, data analysis, or more effective optimization algorithm are needed. Besides, this diagnostic method will be limited by the specific location and the number of the down-lead conductor. The measurement accuracy, detection efficiency and practicality are limited, thus leaving it inconvenient for engineering. An accurate ground network drawing of design is required to obtain the network diagnosis basing on electrical network theory.

\section{ElECTROMAGNETIC FIELD-BASED ANALYSES}

The basic idea is: Inject different frequency sine wave excitation current directly into grounding grids through two deflectors, and then uses magnetic induction coil probe to detect excitation the current has excited on the surface, basing on the principle of electromagnetic induction. The local grounding network conductor's serious corrosion or breakpoint fault happens, as the conductor of the branch cur-rent changes, the distribution of characteristics of the magnetic induction changes, which will provide a reference for the diagnosis.

Literature [25] is based on this method. The author firstly through simulation, focus on the feasibility of diagnostic methods by leading the excitation current of different frequency into the grounding grid and by measuring the surface magnetic flux density distribution characteristics. Next, through comprehensive measurement and analysis of power frequency magnetic fields and transient electromagnetic interference in transformer substations of different voltage levels, the author determines the interference characteristics of substation electromagnetic environment. Finally, through Experiments and the diagnosis applications of actual substation testing, they verify the validity of this diagnostic method and the practicality of the diagnostic techniques. Literature [26] works on the diagnosis of the fracture site by measuring the magnetic field of the ground near the grounding grid. Although this method can detect the conductor fracture, it is difficult to detect the corrosion level of the conductor. Literature [27] works on grounding grid performance analysis and troubleshooting by measuring the surface of the grounding grid potential difference. However, for the grid structure of substation grounding grid, when the grounding grid conductors of local small fracture or corrosion thinning occurs, the surface potential changes are not obvious, thus making detecting defects of the grounding grid conductors by measuring the surface potential difference difficult. Literature [28] uses fuzzy theory to deal with potential data on measurements taken and verified by experiment, achieving good results. Literature [29]-[31], combined with the finite element simulation and the direct excitation power, by measuring the surface magnetic flux density distribution; diagnose grounding grid conductor corrosion thinning and breakpoints.

\section{ELECTROCHEMICAL-BASED METHODS}

The corrosion grounding grid material in the soil occurs is mainly localized corrosion, and the nature of it is galvanic corrosion. Concrete corrosion is of the follow-ing reasons: the macro-cell corrosion caused by soil heterogeneity; micro-cell corrosion caused by carbon steel structure heterogeneity; hydrogen corrosion caused by dc component of the stray current; life activity corrosion caused by microorganisms.

For the electrochemical nature of grounding grid materials corrosion, the corrosion could be described by electrochemical parameters (such as current density). With the metal corrosion dissolution, the number of electrons increases, that is, the power increases. At the same time, there are quantitative relationships between the output power and corrosion (etching amount) during the metal corrosion process [32].

Nowadays, chemical testing of metal corrosion is mainly of the following four categories: [32]-[33]

1) The polarization resistance technique;

2) The transient linear polarization technique

3) Charging curve technique

4) The coulostatic technique.

However, grounding grid corrosion detection based on electrochemical methods remains two unresolved problem. First, the grounding grid is of metal mesh structure, which makes the polarization of the metal area uncertain. In this way, the measuring must be taken to limit. Second, due to the presence of strong stray current in the electrochemical detection method, the signal to noise ratio is very low, possibly even to cover up the real signal. To deal with this, measures must be taken to have the stray current interference filtered.

Literature [34] is based on constant current charging curve technique. By analyzing the curve of value of carbon steel specimen polarization resistance with the specimens' volume buried in the soil area, it determined the effective area of limiting sensor and size of constant current. Literature [35] 
uses electrochemical method for grounding grid corrosion detection. It has developed portable rapid detection system suitable for field applications. It uses limiting probe to solve the problem of the distribution of current. Through the filter and fitting of the data processing it eliminates noise of stray current and soil resistance. By parsing the charging curve it gets the value of polarization resistance $\mathrm{Rp}$ as the characterization of the grounding grid corrosion extent.

In general, research and applications based on the electrochemical method is less. There is a great in-depth study space in this field.

\section{Summary AND OUTLOOK (Ultrasonic Guided WAVE METHOD)}

First, many electric network-based methods use simulation. However, the simulation and actual field-test are different. Actual grounding grid corrosion is both regional and universal. Often in a small area of each branch there are many parts of corrosion, while the simulation is carried out under the assumption that the few parts of the branches have corrosions and the corrosion is severe. Second, when dealing with measurements and node selection, the theory is not perfect. Despite the fact that measurement method of corrosion diagnosis for large and medium-sized substation has been proposed, these methods still need additional measurement of pseudo-fault branch to ensure accuracy, thus plus the tedious work of measuring. Besides such measurement directly affects the efficiency of the test. Third, existing grounding grids corrosion diagnosis program is not written in object-oriented language. Most human-machine interface is unfriendly. The input-output is inconvenient and modeling is cumbersome. Except for that, the main program of the diagnosis is not universal. The operator has to modify its parameters at any time to modify its parameters. All above requires users to learn more about the program, thus adding heavy workload to the system, leaving it lack of promotional value. In addition, the ground network topology may change, the changes will also affect the corrosion diagnosis, and therefore operators need to take some measures to amend the model. The most important, however, is that the methods above cannot accurately locate corrosion point of the grounding grid.

In recent years a fast, long distance, large-scale, relatively low-cost and non-destructive testing method has been developed, namely ultrasonic guided wave inspection method. Ultrasonic guided wave propagates in solids, because of its own characteristics of small attenuation along the propagation path, can overcome the shortcomings of point by point scanning. It can conduct long distances, and wide range defect detection. This detection method can locate defects and reflect the defect levels.

Detection principle of ultrasonic guided wave method: pulse of ultrasonic energy is issued by the probe array. Affected by the role of the waveguide, the magnetic pulse flood the whole root of round steel materials, and spread to the distant. When encountering corrosion or defects, the pulse will be reflected for the material cross section (thickness) changed. The reflected wave returns to and is received by the instrument of the same probe array. By analyzing the reflected signal can detect the location, the size and corrosion condition of the defect.

Literature [38]-[41] studied the propagation and dispersion characteristics of guided waves in cylindrical steel. It introduced the guided wave inspection method in cylindrical. Moreover, it made theoretical and experimental studies of buried anchor detection based on the method of ultrasonic guided wave detection as well as guided wave nondestructive testing of fully bonded resin bolt, which leaves reference value to grounding grid corrosion detection

However, there are many problems to be solved for the based on ultrasonic guided wave method of grounding grid corrosion positioning is immature.

1) Although welding nodes of grounding grid are at fixed location, and the echo signal of corrosion point and the welding nodes are different, technical measures must be taken to distinguish the difference between them.

2) Due to the dispersion characteristics of the guided wave, the appropriate modality must be selected for actual testing.

3) Suitable sensor arrangement must be founded, so as to inhibit of the asymmetric mode signal.

\section{REFERENCES}

[1] V. R. Lawson, "Problems and detection of line anchor and substation ground grid corrosion," IEEE Transactions on Industry Applications, vol. 24, no. 5, pp. 25-31, 1988 •

[2] R. Zeng, J.-L. He, and J. Zou, "Novel method of corrosion diagnosis for grounding grid," in Proc. Conference Record of the IEEE 37th IAS Annual Meeting-Industry Applications Conference, Beijing, 2002, pp. 1120-1126.

[3] B. Zhang, Z.-B. Zhao, and X. Cui, "Diagnosis of breaks in substation's grounding grid by using the Electromagnetic method," IEEE trans. on Magnetic, 2002, vol. 38, no. 2, pp. 473-476.

[4] X.-D. Wu, "Corrosion analysis and countermeasures for grounding grid of $500 \mathrm{kV}$ transmission lines," Corrosion and Protection, 2002, vol. 23 , no. 12 , pp. $545-547$.

[5] D. Ma, "The corrosion analysis and reconstruction suggestions on the grounding grids in Wulashan power plant," Inner Mongolia Electric Power, 2000, vol. 18, no. 3, pp. 38-39.

[6] G.-G. Xie, Power System Over-voltage, China Water Power Press, 1983.

[7] X.-L. Zhang and X.-L. Chen, "The Technique of the Optimization Applied in the Grounding Grid' s Failure Diagnosis of the Power Plant and Substation," High Voltage Engineering, 2000, vol. 26, no. 4, pp. 64-66.

[8] X.-L. Zhang and Q.-Y. Huang, "Fault diagnosis of grounding grid of electric power plants and substations," in Proc. the Chinese Society of Universities for Electric Power System and Automation, 2002, vol. 14, no. 1, pp. 48-51.

[9] X.-H. Xiao, H. Liu, and X.-L. Chen, "Analysis of Theory and Method about the Corrosion as well as the Broken Point of the Grounding Grid," Journal Fault Detection of Grounding Grid of Power System of Chongqing University, 2001, vol. 24, no. 3, pp. 72-75.

[10] Q.-Z. Liu and C.-J. Ding, "Fault Detection of Grounding Grid of Power System," High Voltage Engineering, 2003, vol. 29, no. 6, pp. 19-21.

[11] Y.-G. Liu, Y.-X. Teng, and X.-L. Chen, "A Method For Corrosion Diagnosis Of Grounding Grid," High Voltage Engineering, 2004, vol. 30, no. 6, pp. 19-21.

[12] S. Wang, Y.-G. Liu, and J.-C. You, "Erosion Optimized Diagnosis of Brand Grounding Grid," Journal of Chongqing University, 2006, vol. 29, no. 8, pp. 33-35.

[13] Y.-G. Liu, S. Wang, and J.-H. Tian, "A corrosion diagnosis method for optimized measurement of grounding grids," Journal of Chongqing University, 2008, vol. 31, no. 11, pp. 1303-1306.

[14] J. Hu, W.-M. Sun, and J.-X. Yao, "Novel Method of Corrosion Diagnosis for Grounding Grid," in Proc. of Power Conference 2000 vol. 3, pp. 1365-1370 
[15] Y.-G. Liu, Y.-X. Teng, and X.-L. Chen, “A Method for State Diagnosis of Grounding Grid," Journal of Chongqing University(Natural Science Edition), 2004, vol. 27, no. 2, pp. 92-95.

[16] X.-B. Jiang, "A new method of fault diagnosis for grounding grids," Journal of Fuzhou University, 2005, vol. 33, no. 6, pp. 749-752.

[17] J. Liu, J.-X. Wang, and S. Wang, "An improved algorithm of corrosion diagnosis for grounding grids \& its evaluation," in Proc. the Csee, 2005, vol. 25 , no. 3, pp. 71-77.

[18] W.-W. Huang, X.-S. Wen, and Z.-G. Zhu, "Development of the Diagnosis Software System for the Corrosion and the Broken Point of the Grounding Grid," High Voltage Engineering, 2005, vol. 31, no. 7, pp. $42-45$.

[19] L. Guo, "A Example Of Grounding Device Corrosion Fault Diagnosis," Northwest China Electric Power, 2003, pp. 6.

[20] The development of Substation ground grid defects diagnostic system, Shanxi Electric Power Research Institute Technical Report, XDY/KF-09-02-2004, Shaanxi Electric Power Research Institute, 2006.

[21] Y.-X. Teng, A Method for Corrosion Diagnosis of Grounding Grid, Chongqing University, 2004

[22] J. Liu, S.-Q. Wang, and Z.-Z. Li, "Testability of Grounding Grids Corrosion Diagnosis," High Voltage Engineering, 2008, vol. 34, no. 1 , pp. 64-69.

[23] Y.-F. Ni, J. Liu, and S.-Q. Wang, "Principle and application of conductor corrosion diagnosis for grounding grids," Journal of $X i$ " an University of Science and Technology, 2008, vol. 28, no. 2, pp. 318-322.

[24] S. Wang, Y.-G. Liu, and J.-C. You, "Erosion Optimized Diagnosis of Brand Grounding Grid," Journal of Chongqing University, 2006, vol. 29, no. 8, pp. 33-35.

[25] Y. Liu, "Research of Method and Technology on the Defect Diagnosis of Grounding Grids in Substations," North China Electric Power University, 2008.

[26] F. P. Dawalibi, "Electromagnetic Fields Generated by Overhead and Buried Short Conductors Part 2-Ground Conductor," IEEE Trans on Power Delivery, 1986, PWRD-1, pp. 112-119.

[27] B. Zhang, Z.-Bin. Zhao, X. Cui et al., "Diagnosis of Breaks in Substation's Grounding Grid by Using the Electromagnetic Method," IEEE Trans on Magnetics, 2002, vol. 38, no. 2, pp. 473-476.

[28] P.-H. Zhang, J.-J. He, and X.-G. Yin, "Method of Grounding Grid Fault Diagnosis Based on Fuzzy Theory," High Voltage Apparatus, 2011, vol. 47 , no. 5 , pp. $54-58$.

[29] Y, Liu, X. Cui, and Z.-B. Zhao, "Method of Corrosion Diagnosis of Substation's Grounding Grids Based on Electromagnetic Induction Theory," in Proc. of the CSEE, 2009, vol. 29, no. 4, pp. 91-102.

[30] Y. Liu, X. Cui, and Z.-B. Zhao, "Method for Diagnosing the Conductor Broken Point of Grounding Grids in Substation Based on Measuring the Magnetic Induction Intensity," High Voltage Engineering, 2008, vol. 34, no. 7, pp. 1389-1394.

[31] Y. Liu, X. Cui, and Z.-B. Zhao, "Method of Structure Estimation and Fault Diagnosis of Substations' Grounding Grids," in Proc. of the CSEE, 2010, vol. 30, no. 24, pp. 113-117.

[32] X.-W. Hu and C.-W, "Xu,Research on Corrosion and Protection of Grounding Grid," Hubei Electric Power, 2002, vol. 26, no. 3, pp. 31-34.

[33] X.-S. Wang, B. Tian, and S.-Z. Song, "Study on the electrochemical sensing system for monitoring/detecting marine steel corrosion," Journal of Chinese Society For Corrosion and Protection, 2001, vol. 21, no. 3, pp. 182-187.

[34] X.-L. Zhang, N. Yang, and P. Luo, "Corrosion Rate Measurement of Carbon Steel Grounding Grid With Galvanostatic Charging Curve Technique," in Proc. the CSEE, 2009, vol. 29, no. 4, pp. 97-103.
[35] L. Han, S.-Z. Song, and X.-L. Zhang, "Development and application of a portable electrochemical device for diagnose of grounding grid corrosion," Corrosion Science and Protection Technology, 2009, vol. 21, no. 3, pp. 337-340.

[36] G. Sharick, Grounding and Bonding, Revised Second Edition, 1999.

[37] A. J. Wakeling and A. McKeon, "Fault diagnosis in analogue electronics without fault models or prior experience," IEE Colloquium on Intelligent Fault Diagnosis - Part 2:Model-Based Techniques, vol. 26, Feb. 1992, pp. $61-64$.

[38] B. Wu, L.-T. Li, and X.-Y. Wang, "Non-Destructive Test Of A Surface defect on a steel bar based on ultrasonic guided wave techniques," Engineering Mechanics, 2003, vol. 20, no. 5, pp. 149-154.

[39] C.-F. He, Y.-X. Sun, and B. Wu, "Application of ultrasonic guided waves technology to inspection of bolt embedded in soils," Chinese Journal of Geotechnical Engineering, 2006, vol. 28, no. 9, pp. 1144-1147.

[40] M. D. Beard, Guided wave inspection of embedded cylindrical structures, London: Imperial College, 2002.

[41] J.-Y. Cui, Y.-X. Sun, and C.-F. He, "Application of low frequency ultrasonic guided waves on inspection of full-length-bonding resin bolt," Engineering Mechanics, 2010, vol. 27, no. 3, pp. 240-245.

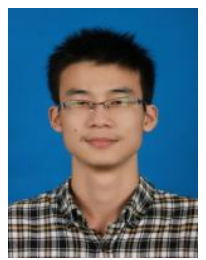

Wenguang Chen was born in December 1990. He is an undergraduate from Wuhan University. one of the member of " the study on the orientation of grounding grip corrosion based on ultrasonic guided wave "which is one of The National College Students' Innovative Training Project.

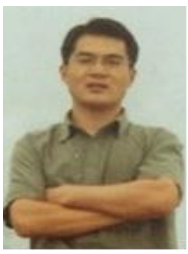

Ruyu Bi was born in February 1991. He is an undergraduate from School of Electrical Engineering Wuhan University, major in Electrical engineering and automation.

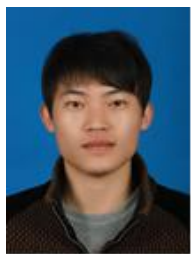

Jian Wang is an undergraduate from Wuhan University. one of the member of " the study on the orientation of grounding grip corrosion based on ultrasonic guided wave "which is one of The National College Students' Innovative Training Project .

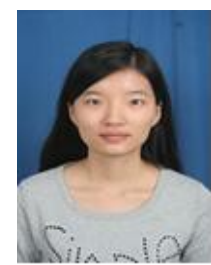

Hougui Chen is an associate professor of Wuhan University, who is a director of Hubei Society of Mechanical Engineering. $\mathrm{He}$ is working in non-destructive testing for years. 